\title{
COMPLICACIÓN BUCAL TARDÍA, POSTERIOR A PALATOPLASTIA EN FISURA LABIOPALATINA BILATERAL: RELATO DE CASO CLÍNICO
}

\begin{abstract}
Cirujano Dentista / Ortodoncista. Universidad Mayor de San Andrés, La Paz, Bolivia / Hospital de Rehabilitación de Anomalías Craneofaciales - Universidad de São Paulo, Bauru, Brasil.

2 Cirujano Dentista Odontopediatra. Hospital de Rehabilitación de Anomalías Craneofaciales - Universidad de São Paulo, Brasil.

${ }^{3}$ Cirujano Dentista, Docente del Departamento de Odontopediatria y Salud Colectiva del Hospital de Rehabilitación de Anomalías Craneofaciales - Universidad de São Paulo, Bauru, Brasil.

${ }^{4}$ Jefe del Departamento de Odontopediatria y Salud Colectiva del Hospital de Rehabilitación de Anomalías Craneofaciales - Universidad de São Paulo, Bauru, Brasil.
\end{abstract}

Correspondencia a: Ingrid Ivanna Huayta Aguirre Correo electrónico: ivannahuayta@gmail.com

Telefono: +59178885395

ORCID: https://orcid.org/00000002-7287-9291

Palabras clave: Fisura palatina Procedimientos quirúrgicos reconstructivos. Complicaciones postoperatorias.

Keywords: Cleft palate. Reconstructive surgical procedures. Postoperative complications.

Procedencia y arbitraje: no comisionado, sometido a arbitraje externo.

Recibido para publicación: 20 de abril 2020

Aceptado para publicación: 21 de abril 2020

Citar como:

Huayta Aguirre II, Dias de Sena M, Costa B, Dalben GDS Complicación bucal tardía, posterior a palatoplastia en fisura labiopalatina bilateral: relato de caso clínico. Rev Cient Cienc Med. 2021; 24(1): 180-186

\section{LATE ORAL COMPLICATION AFTER PALATOPLASTY IN BILATERAL CLEFT LIP AND PALATE: CLINICAL CASE REPORT}

\author{
Dra.Ingrid Ivanna Huayta Aguirre ${ }^{1}$, Dra.Marina Dias de Sena², Dra.Beatriz Costa ${ }^{3}$, \\ Dra.Gisele da Silva Dalben ${ }^{4}$.
}

\section{RESUMEN}

La fisura labiopalatina bilateral es una anomalía craneofacial común y multifactorial, pudiendo estar asociada a factores genéticos y ambientales. El reparo de una fisura palatina requiere de varias intervenciones quirúrgicas y no quirúrgicas por parte de un equipo multidisciplinar. El perfeccionamiento de las técnicas quirúrgicas reduce la prevalencia de complicaciones intra y posoperatorias. Como en cualquier cirugía, la palatoplastia presenta algunos riesgos inherentes. Evaluar el resultado quirúrgico es un desafío debido a los diversos factores que lo afectan; incluyendo el tipo de fisura y su extensión, peculiaridades del paciente, experiencia del cirujano, técnica adoptada y manejo posoperatorio. El presente relato de caso describe, e ilustra mediante fotografías, los datos clínicos encontrados posterior a palatoplastia de una paciente con fisura labiopalatina bilateral, así como sus complicaciones bucales y el plan de tratamiento odontológico propuesto para otorgar función y estética en el área afectada.

\section{ABSTRACT}

Cleft lip and lor palate is a common and multifactorial craniofacial anomaly, and may be associated with genetic and environmental factors. The repair of cleft palate often requires several surgical and non-surgical interventions by a multidisciplinary team. The improvement of surgical techniques reduced the prevalence of intra- and postoperative complications. However, as for any surgery, palatoplasty presents some inherent risks. Evaluating the surgical outcome is challenging due to the several influencing factors, including the cleft type and extent, patient's peculiarities, surgeon experience, technique adopted and postoperative management. The present case report described, and illustrated by images, a bilateral cleft lip and palate individual clinical data found posterior to palatal surgery, as well as its oral complications and proposed dental treatment plan to grant function and esthetics to the affected area.

\section{INTRODUCCIÓN}

a fisura labiopalatina es la segunda - anomalía congénita más común en el ser humano con una incidencia de 1 cada 650700 nacidos vivos en el Brasil ${ }^{1,4}$, de etiología multifactorial, requiere de la intervención de un equipo multidisciplinar para conseguir la rehabilitación de los individuos afectados ${ }^{2}$. Puede ser tratada quirúrgicamente con éxito, especialmente si estas intervenciones son realizadas poco tiempo después del nacimiento o en la primera infancia ${ }^{1}$.

Sin embargo, las complicaciones quirúrgicas pueden ocurrir e incluyen tanto eventos postoperatorios inmediatos como problemas de calidad de vida a largo plazo, dependiendo de la complejidad de los procedimientos y de las características individuales del paciente ${ }^{3}$.

A partir de la identificación de los factores de riesgo, se deben desarrollar protocolos para mejorar los cuidados con la fisura y disminuir la frecuencia de esos eventos adversos $^{3}$. Los mismos que, por su ubicación anatómica, se verán principalmente concentrados en el maxilar superior afectando el sistema estomatognático. La discontinuidad de la estructura maxilar y la 
fragilidad de los segmentos maxilares ante las cirugías plásticas primarias representan un problema odontológico de importancia a ser tratado ${ }^{2}$ hasta el final del crecimiento óseo del paciente principalmente. La adición de algún tipo de complicación quirúrgica incrementará el grado de complejidad de la atención especializada repercutiendo en el incremento de procedimientos especializados y el de tiempo de tratamiento haciendo que la rehabilitación sea más costosa e incrementado el burden of care $^{5}$.

El objetivo de este trabajo es relatar un caso de complicación odontológica tardía posterior a la realización de la cirugía de palatoplastia secundaria, sus consecuencias y las medidas tomadas, con el fin de preservar la forma y la función de las estructuras bucales.

\section{PRESENTACIÓN DEL CASO}

Paciente femenino de 8 años de edad, se presentó a la clínica de Odontopediatría del Hospital de Rehabilitación de Anomalías Craniofaciales (HRAC), para la realización del examen clínico de rutina y profilaxis profesional. La historia médica de la paciente revela que la misma poseía fisura labiopalatina del tipo transforamen incisivo bilateral (clasificación de Spina), la misma que fue reconstruida quirúrgicamente.

Fueron realizados tres procedimientos quirúrgicos hasta el momento del examen clínico, siendo ellos: corrección de labio (queiloplastia primaria), corrección del paladar (palatoplastia primaria) y cirugía para la corrección de fistula buconasal persistente ubicada posterior a la premaxila. Las mencionadas cirugías fueron realizadas, respectivamente, a los dos, tres y seis años de edad de la paciente. Las mismas que se ejecutaron fuera de tiempo en relación al protocolo usado por la institución (ver tabla 1).

Evaluando la historia clínica de la paciente, se pudo evidenciar que previamente a la realización de la cirugía de corrección de fistula buconasal, la paciente fue enviada al sector de Odontopediatría para evaluación de retención prolongada de incisivo central superior izquierdo deciduo (figura 1). Fue realizada una radiografía periapical de la región, y se constató que a pesar de que ambos incisivos centrales superiores permanentes ya estaban erupcionados, el diente 61 había sufrido el proceso de rizólisis, optándose, en aquel momento, por su manutención considerando el aspecto estético y preservación del caso. La evaluación clínica y fotográfica constató la presencia de una premaxila robusta abarcando cuatro dientes erupcionados además de diastemas de consideración entre todos estos. La visión lateral y submentoniana muestran una premaxila prominente sagitalmente con consecuente overjet dentario aumentado.

La presente evaluación fue realizada dos años después de la última cirugía plástica a la que la paciente fue sometida (palatoplastia para corrección de fístula buconasal persistente) y durante el examen clínico se observó la ausencia de los elementos dentarios 12, 61 y 21 (figura 2), así como la reducción acentuada del volumen y proyección sagital de la premaxila además de la perdida ósea severa en la región palatina del diente 11. Ninguna información acerca de complicaciones intraoperatorias fue registrada en la Historia Clínica de la paciente.

Posterior a una nueva radiografía periapical en el área, realizada en el mismo día de la atención, fue constatado que el incisivo central superior derecho permanente estaba periodontalmente comprometido y

Tabla 1. Protocolo de intervenciones quirúrgicas del Hospital de Rehabilitación de Anomalías Craneofaciales - Universidad de São Paulo (HRAC-USP).

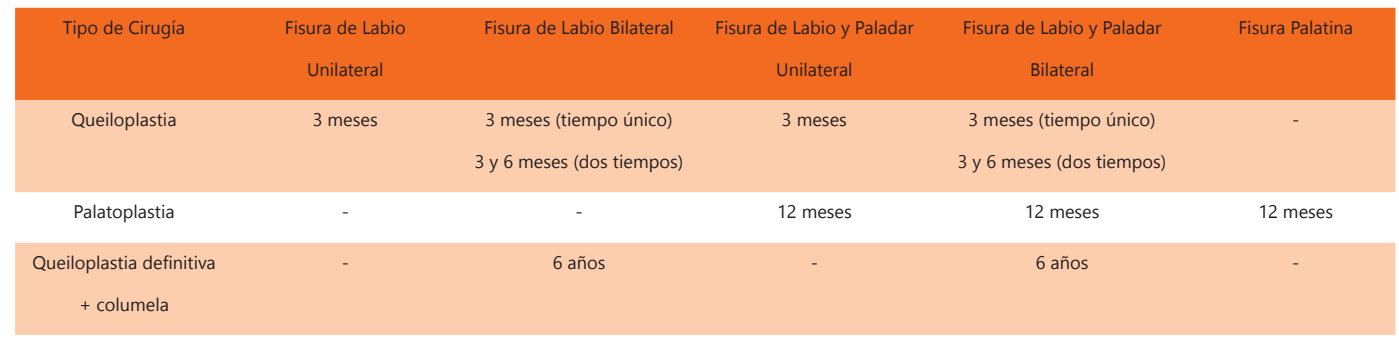

Fuente: Trindade IEK, Silva Filho OG coord. Fissuras labiopalatinas, uma abordagem interdisciplinar 


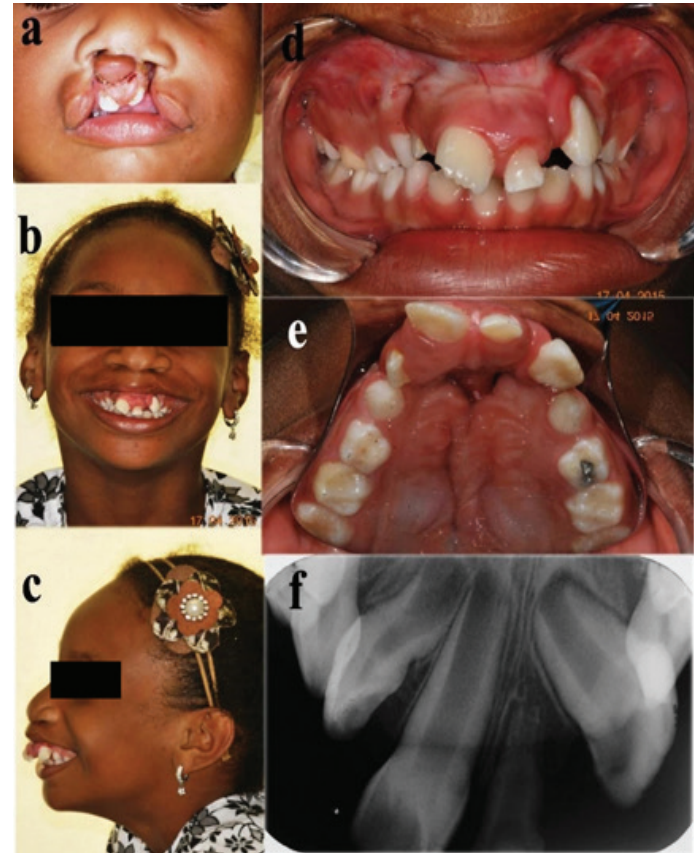

Figura 1: Características faciales, intraorales y radiográficas PREVIAS a palatoplastia secundaria (paciente con 6 años de edad). A, características faciales previa a cirugías plásticas primarias. $B-C$ muestran las características faciales donde la proyección de la premaxila es evidente. $D$-E, relación interarcos y características del maxilar previo a la cirugía muestran una premaxila robusta albergando 4 dientes erupcionados $F$, radiografia periapical mostrando los dientes de la premaxila sin alteraciones radiculares ni óseas, salvo la leve reabsorción periapical del diente deciduo.

condenado debido a la falta de soporte óseo, lo que imposibilitaba su adecuada función y alteraba grandemente la estética, siendo necesaria su exodoncia. Es importante mencionar que debido a que el HRAC recibe a pacientes con fisura labiopalatina de todo Brasil, los procedimientos odontológicos se realizan en cortos periodos de tiempo considerando que la gran mayoría de los pacientes viven en regiones alejadas, como es el caso del presente caso por tanto, se realizaron todos los procedimientos clínicos en un único día.

Previamente a la extracción, se optó por realizar la toma de impresiones de los arcos maxilar y mandibular de la paciente, para que ese mismo día sea confeccionada e instalada una placa superior removible con dientes artificiales, en la tentativa de preservar la forma y la función de las estructuras bucales

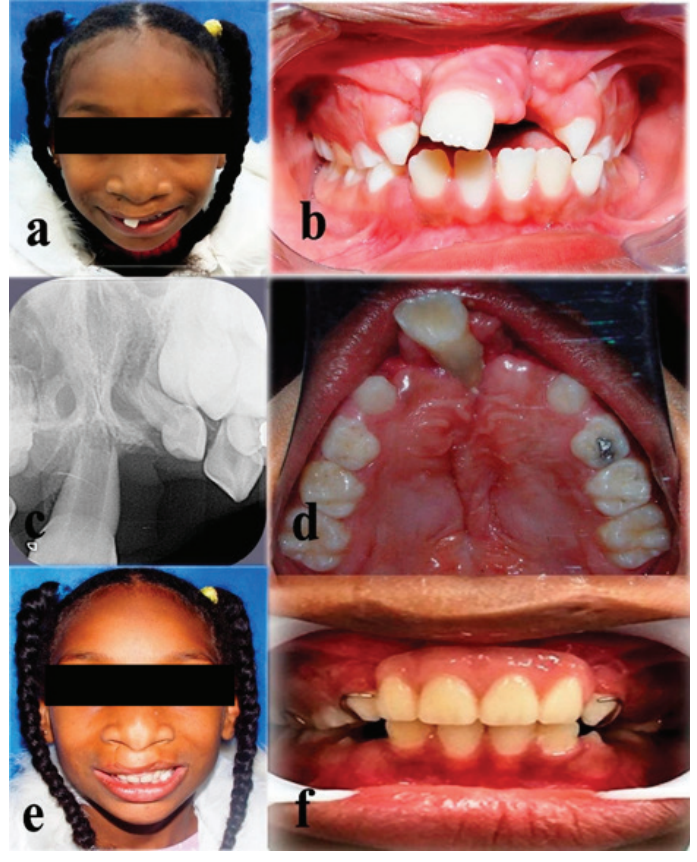

Figura 2: Características faciales, intraorales y radiográficas POSTERIORES a palatoplastia secundaria (paciente con 8 años de edad). A-B-C-D, muestran los resultados estéticos, oclusales y radiográficos dos años después de la intervención quirúrgica para tratamiento de fistula buconasal persistente, se observa una premaxila reducida drásticamente en volumen y exposición radicular por palatino del diente 11. E-F, características faciales y oclusales posteriores a la exodoncia del diente 11 e instalación inmediata de la placa removible con dientes artificiales.

y minimizar los efectos psicológicos causados por la pérdida dentaria.

La impresión de ambos arcos fue realizada con alginato convencional seguida del registro de la mordida en cera, inmediatamente las cubetas con las impresiones fueron conducidos al laboratorio de prótesis para la obtención de los registros en positivo de los modelos dentarios usando yeso extra duro en su fabricación. La exodoncia fue realizada inmediatamente después de la toma de impresiones, sobre anestesia local y sin complicaciones. Una hora después de la toma de impresiones, los modelos retornan a la clínica de Odontopediatria para evaluación y diseño de la prótesis, una vez concertado el tipo de aparato a ser confeccionado y ser realizada la exodoncia en el modelo de la pieza dentaria 11, se dan las orientaciones al protesista encargado. Se determina la 
realización de una prótesis removible con ganchos circunferenciales en caninos y segundos molares deciduos, así como la adición de cuatro dientes de acrílico en la región anterior para reemplazar la ausencia de los cuatro incisivos superiores. Algunas horas más tarde, la paciente retornó a la clínica para la instalación y ajustes de la prótesis o placa removible. Fue necesario realizar un leve desgaste con fresas de laboratorio en palatino de los dientes de acrílico de la placa para poder obtener una adecuada relación oclusal.

Cuatro meses después, la paciente retornó para el primer control. Se verificó que la región donde fue realizada la extracción estaba debidamente cicatrizada y la placa continuaba bien adaptada, sin embargo, existía necesidad de material resinoso en su porción anterior, probablemente debido al mayor alivio que fue necesario en esta región debido a la realización de la exodoncia en el mismo día de confección de la placa y a la reducción del tamaño de la premaxila al no presentarse más dientes en esta región. Se optó entonces por su cambio exclusivamente por motivos estéticos, se siguieron los mismos lineamientos que fueron usados con la primera placa removible, siendo realizados nuevamente la impresión de los arcos dentario, confección de la misma con la única diferencia que en esta oportunidad para mejorar la adaptación se adaptaron ganchos circunferenciales en los caninos permanentes, primeros premolares y en los primeros molares permanentes, ya que la paciente no se retira la prótesis para su alimentación. Finalmente se realizó la instalación de la placa removible el mismo día y fueron realizados los ajustes, que en este momento contemplaron el desgaste en la región aledaña a los caninos permanentes .

Después de 3 años de seguimiento y con la paciente con 10 años, pudo observarse una premaxila reducida en volumen y nivelada en relación a los segmentos maxilares (figura 3), el uso de la placa removible es continuo y de gran aceptación por parte de la paciente y los responsables, quienes vieron en ella una medida adecuada para salvaguardar la estética y función del sistema estomatognático.

\section{DISCUSIÓN}

Hasta el momento, muchas técnicas
quirúrgicas fueron desarrolladas e

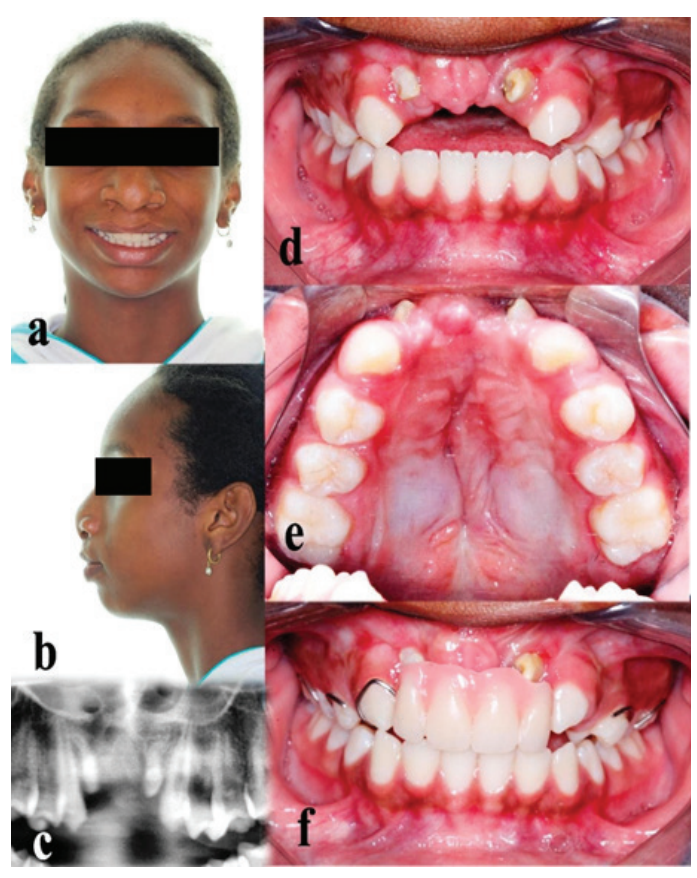

Figura 3: Características faciales e intraorales después de 3 años de seguimiento (paciente con 11 años de edad). A-B, características extraorales armónicas. $C$, segmento de radiografía panorámica mostrando dos dientes supranumerarios erupcionados en los segmentos maxilares. D-E, fotografias intrabucales evidenciando la ausencia casi total de la premaxila tanto en visión frontal como oclusal. F, adaptación de placa removible provisional con dientes artificiales.

implementadas con la intención de mejorar los resultados de las cirugías de fisura labiopalatina, que básicamente es devolver la unidad a los tejidos maxilares sin afectar significativamente su crecimiento y desarrollo. Las complicaciones relacionadas a estos procedimientos quirúrgicos se intentan evitar, prevenir o disminuir, sin embargo, esto no es siempre posible para el equipo rehabilitador y especialmente para el cirujano, siendo que la complicación más común es la del tipo respiratorio posterior al reparo del labio y paladar?.

La fistula buco-nasal es una de las complicaciones de palato-plastia más comunes a largo plazo en individuos con fisura labio-palatina operados. Su prevalencia puede variar entre $3 \%$ a $50 \%$, pero la mayoría de los estudios recientes muestran una frecuencia de $4.9 \%^{8-9}$. Su ocurrencia puede estar relacionada a la técnica quirúrgica utilizada ${ }^{8}$, experiencia del cirujano, extensión de la fisura, mala cicatrización de la herida quirúrgica, tensión de los tejidos o infección local ${ }^{10}$, y la conducta 
puede variar desde la observación hasta la indicación de una cirugía secundaria" ${ }^{11}$.

En el caso presentado, la paciente necesitó de una cirugía para la corrección de una fístula buco-nasal persistente posterior a la palatoplastia primaria, que fue realizada a los seis años de edad, momento en que es reevaluada la necesidad de cirugías secundarias. Siguiendo las informaciones colectadas en la historia clínica médica, no existió ningún tipo de complicación trans o posoperatoria que justificase posteriormente la pérdida de tres piezas dentarias en la premaxila y la existencia de pérdida ósea extensa observada en la región palatina del incisivo central derecho, lo que conllevó a su consecuente exodoncia.

En la literatura no fue encontrado un caso similar, sin embargo, se sabe que la necrosis de tejido posterior a la reparación del paladar es un problema poco frecuente, pero significativo, que puede ser atribuido a diferentes causas como compresión, tensión o sección del pedículo, trombosis vascular, sangrado y hematoma, y lesión quirúrgica durante la intervención quirúrgica. Pocos estudios relatan esta complicación y su prevalencia es todavía desconocida ${ }^{12}$.

Entre las complicaciones peri-operatorias y posoperatorias Nwoku, citado por Taiwo ${ }^{13}$, señala la posibilidad de existencia de daño a los centros de crecimiento óseo y lesión de gérmenes dentarios especialmente en niños. Sin embargo, el caso presentado no presenta la lesión de algún germen dentario específicamente, sino más bien la necesidad de extracción de los dientes 12,21 y 61 presumiblemente por parte del cirujano durante el acto operatorio para que la fistula pudiese ser adecuadamente tratada o posterior a algún tipo de trauma en la premaxila, que encajaría mejor con la descripción de los sucesos posteriores. La prevalencia del trauma oral en niños con fisuras labio-palatinas bilateral es alta, entre $47 \%$ y $56 \%{ }^{14}$, debido a la marcada exposición de la premaxila a traumas, como consecuencia de su proyección sagital. Sin embargo, debido a que no se encuentra información al respecto en la historia clínica y los responsables de la paciente declaran que no existió histórico de traumas dentales y/o faciales, las razones de la pérdida dentaria y ósea en la premaxila del caso presentado son aún desconocidas.
Es necesario mencionar la importancia de la pieza dentaria en el crecimiento y desarrollo del hueso alveolar, ya que la formación de hueso alveolar está estrictamente coordinada con la formación dentaria desde la etapa prenatal $^{15}$ y la perdida temprana de éstos producirá la reabsorción de las tablas óseas adyacentes a los mismos e incluso reducir considerablemente el volumen de la premaxila como en el caso presentado. Además, a medida en que los dientes deciduos exfolian y los dientes permanentes erupcionan, ocurre una secuencia de eventos fisiológicos que resultan en una oclusión funcional, estética y estable. Cuando esa secuencia de sucesos es interrumpida, como ocurrió en el actual caso clínico, procedimientos rehabilitadores pueden ser necesarios para traer de vuelta la armonía de los componentes estéticos y funcionales de la dentición en desarrollo. El tratamiento de la perdida precoz de dientes permanentes en pacientes jóvenes es complicado debido a la dificultad en realizar procedimientos restauradores definitivos en un perfil dental que está en constante cambio ${ }^{16}$. La colocación de una prótesis fija o implante para la substitución dentaria raramente es la mejor opción de tratamiento debido al crecimiento cráneo-facial incompleto. Si es posible, la sustitución de los elementos perdidos debe ser hecha por medio de una placa removible confeccionada con base de resina acrílica y dientes artificiales que permita ajustes y libertad de crecimiento, que requiere de áreas disponibles para la colocación de ganchos de retención y cambios frecuentes. Se trata por en tanto, de una intervención en largo plazo y que permanece hasta la finalización del crecimiento óseo, cuando una medida permanente podrá ser ejecutada.

De esta forma, el objetivo del tratamiento de otorgar un adecuado resultado estético y funcional, que fuese bien tolerada por la paciente y que también pudiese ser modificada para permitir la erupción dentaria en los segmentos maxilares fue llevado a cabo exitosamente mediante la confección e instalación de una placa removible con dientes artificiales. Deberán ser realizados cambios sucesivos, en un intervalo de tiempo de aproximadamente cuatro meses, cuando la misma se desadapte y su uso discontinuado cuando la paciente inicie su tratamiento 
ortodóntico/ortopédico para finalizar con la rehabilitación protética final al término del crecimiento óseo.

Después de 3 años de seguimiento y en uso continuo de la placa removible pudo observarse una premaxila muy reducida en volumen y nivelada en relación a los segmentos maxilares, esta relación podría verse alterada en el futuro debido a la existencia de remanente de crecimiento óseo a los diez años de edad. Podría establecerse una relación maxilo-mandibular negativa que conllevaría a la realización de procedimientos quirúrgicos invasivos para salvaguardar la función y estética de la misma. Todo esto en función de la existencia de complicaciones quirúrgicas que talvez podrían haber sido prevenidos y/o evitados.

\section{CONCLUSIÓN}

La frecuencia de complicaciones posterior a la palatoplastia compromete los objetivos de la cirugía y altera el gerenciamiento futuro del caso por el equipo rehabilitador multidisciplinar. Al identificar los factores de riesgo, protocolos basados en evidencia deben ser desarrollados para mejorar los resultados y disminuir los eventos adversos. Así también, controles periódicos por parte del equipo de trabajo, aseguran la continuidad de los cuidados y una mejor evaluación de las complicaciones, que esos pacientes puedan llegar a tener incluso posterior a la corrección quirúrgica de su problema. 
1. Sousa GF, Roncalli AG. Orofacial celfts in Brazil and surgical rehabilitation under the Brazilian National Health System. Braz Oral Res [Internet]. 2017 [citado 20 Ene 2020]; 31(23). Disponible en: http://www. scielo.6r/scielo. php? script $=$ sci_arttextcipid $=$ S180683242017000100222 \&'ing=en. Epub Mar 30, 2017. https://doi.org/10.1590/1807-31076or-2017. voß31.0023

2. Freitas JA. Rehabilitative treatment of cleft lip and palate: experience of the Hospital for Rehabilitation of Craniofacial Anomalies /USP (HRAC/USP)-Part 1: overall aspects. J Appl Oral Sci. 2012; 20(1): 9-15.

3. Cervenka B, Setabutr D, Rubinstein BK. Surgical repair of the cleft palate. Oper Tech Otolayngol Head Neck Surg. 2015; 26:121-6.

4. Freitas JA, Garib DG, Oliveira $M$, et al. Rehabilitative treatment of cleft lip and palate: experience of the Hospital for Rehabilitation of Craniofacial Anomalies-USP (HRAC-USP)—part 2: pediatric dentistry and orthodontics. J Appl Oral Sci. 2012;20(2):268-81

5. Alberconi TF, Siqueira GLC, Sathler R, et al. Assessment of orthodontic burden of care in patients with unilateral complete cleft lip and palate. Cleft Palate Craniofac J. 2018; 55(1): 7478.

6. Bertier CE, Trindade IEK, Silva Filho OG. Cirurgias primárias de lábio e palato. En: Trindade IEK, Silva Filho OG coord. Fissuras labiopalatinas, uma abordagem interdisciplinar.1ra Ed. Brasil: Livraria editora Santos; 2007. p. 73-86.

7. Paine KM, Tahiri Y, Wes AM, Wink JD, Fischer JP, Gelder CA, et al. An Assessment of 30-Day Complications in Primary Cleft Lip Repair: A Review of the 2012 ACS NSQIP Pediatric. Cleft Palate Craniofac J. 2016; 53:283-9.

8. Tse RW, Siebold B. Cleft palate repair: description of an approach, its evolution and analysis of postoperative fistulas. Plas reconstr Surg. 2018; 141(5):1201-14

9. Bykowski MR, Naran S, Winger DG, Losee JE. The rate of oronasal fistula following primary cleft palate surgery: a metanalysis. Cleft Palate Craniofac J. 2015; 52(4):e81-7

10. Li F, Wang HT, Chen YY, Wu WL, Liu JY, Hao JS et al. Cleft relapse and oronasal fistula after Furlow palatoplasty in infants with cleft palate: incidence and risk factors. Int J Oral
Maxillofac Surg [Internet]. 2017 [Consultado 22 Ene 2020];46(3):275-80. Disponible en: https://www.sciencedirect.com/science/article/pii/ S0901502716302478?via\%3Difu6

11. Woo AS. Evidence-based medicine: cleft palate. Plast Reconstr Surg. [Internet]. 2017 [Consultado 10 Dec 2019]; 139(1):191203. Disponible en: https://insights.ovid.com/ pubmed? pmid $=28027255$

12. Rossell-Perry P. Flap necrosis after palatplasty in patients with cleft palate. Biomed Res Int [Internet]. 2015 [Consultado 15 Feb 2017]; 2015:516375. Disponible en: http:// dx.doi.org/10.1155/2015/516375

13. Taiwo OA, Adeyemi OM, Adeyemo WL, Ogunlewe MO, Ladeinde LA.Surgical outcome and complications following cleft lip and palate repair in a teaching hospital in Nigeria. Afr J Paediatr Surg. [Internet]. 2013 [Consultado 4 Mar 2020]; 10(4):345-59. Disponible en: https:// www.researchgate.net/publication/259956621_ Surgical_outcome_and_complications_following_ cleft_lip_and_palate_repair_in_a_teaching_ hospital_in_Nigeria

14. Da Silva JYB, Aranha AMF, Peixoto V, Costa B, Gomide MR. Prevalence of oral trauma in children with bilateral clefts. Dental Traumatology. 2005; 21:9-13

15. Kjaer I, Bgheri A. Prenatal development of the alveolar bone of human deciduous incisors and canines. J Dent Res. [Internet]. 1999 [Consultado 10 Dec 2019]; 78(2):667-72. Disponible en: https://journals.sagepu6.com/doi/pd f/10.1177/00220345990780020601

16. Rubel B, Hill EE. Replacement of maxillary permanent central incisors lost due to trauma in the mixed-dentition. Tex Dent J. 2014 Aug;131(8):582-5. 